\title{
CENTRAL LIMIT THEOREM FOR THE SOLUTION OF THE MULTIDIMENSIONAL BURGERS EQUATION WITH RANDOM DATA
}

\author{
A.V. Bulinskii \\ Moscow State University, Department of Mechanics and Mathematics \\ 119899 Moscow, Russia
}

\begin{abstract}
For multidimensional Burgers equation the potential case is considered. This model for nonlinear diffusion is studied for shot-noise random fields as initial potentials. For the solution $\vec{v}$ the limiting behavior of the field $\vec{v}(\alpha t, a \sqrt{t})$ is investigated as $t \rightarrow \infty\left(\alpha \in \mathbf{R}_{+}, a \in \mathbf{R}^{d}\right)$.
\end{abstract}

The objective of this paper is to apply the Central Limit Theorem for random fields defined on a $d$-dimensional lattice $\mathbf{Z}^{d}$ to investigate the asymptotical behavior of some integral functionals depending on random fields defined on $\mathbf{R}^{d}$. A nontrivial example of such nonlinear vector valued functionals, arising in certain physical problems, is provided by the solution of the Cauchy problem for the multidimensional Burgers equation

$$
\begin{aligned}
\frac{\partial \vec{v}}{\partial t}+(\vec{v}, \nabla) \vec{v} & =\nu \Delta \vec{v}, \quad x \in \mathbf{R}^{d}, t>0, \\
\vec{v}(0, x) & =\vec{v}_{0}(x),
\end{aligned}
$$

with random initial data. Here $(\cdot, \cdot)$ stands for a scalar product in $\mathbf{R}^{d}$, and $\nu$ is some positive constant (viscosity coefficient). We are interested in the behavior of the solution for large values of the time parameter $t$. This equation has been widely used to model nonlinear diffusion, especially in the cases $d=1,2,3$. In particular it has been applied in astro-physical scenarios of the early Universe, see [1], [8], [12]. The present paper continues and in some aspects extends the joint work with S.A. Molchanov [6], see also [3], [4].

One advantage of using Burgers equation is the existence of an analytic solution in a very important (from the cosmological point of view) case of motion of potential type, i.e., $\vec{v}(t, x)=\nabla \Phi(t, x)$ and $\Phi_{0}$ being the potential of $\vec{v}_{0}$. The well-known Hopf-Cole substitution leads to an explicit formula

$$
\begin{aligned}
\vec{v}(t, x)= & \int_{\mathbf{R}^{d}} \frac{x-y}{t} \exp \left\{-\frac{1}{2 \nu}\left(\Phi_{0}(y)+\frac{|x-y|^{2}}{2 t}\right)\right\} d y . \\
& \cdot\left(\int_{\mathbf{R}^{d}} \exp \left\{-\frac{1}{2 \nu}\left(\Phi_{0}(y)+\frac{|x-y|^{2}}{2 t}\right)\right\} d y\right)^{-1}
\end{aligned}
$$


here $|\cdot|$ denotes the Euclidean norm, and the integral of the vector-valued function is taken in the usual sense.

In order to indicate the dependence of the solution on $\Phi_{0}$ and $\nu$ we write $\vec{v}\left(t, x ; \Phi_{0}, \nu\right)$. After a transformation of variables we examine the asymptotic behavior of the vector field

$$
Z_{t}\left(\alpha, a ; \Phi_{0}, \nu\right)=\vec{v}\left(\alpha t, a \sqrt{t} ; \Phi_{0}, \nu\right), \quad \alpha \in \mathbf{R}_{+}, a \in \mathbf{R}^{d} \text { as } t \rightarrow \infty .
$$

Since for any $\nu>0$

$$
Z_{t}\left(\alpha, a ; \Phi_{0}, \nu\right)=\sqrt{2 \nu} Z_{t}\left(\alpha, a / \sqrt{2 \nu} ; \Phi_{0}(\cdot \sqrt{2 \nu}) / 2 \nu, \frac{1}{2}\right),
$$

we can consider, without loss of generality, just the case $\nu=\frac{1}{2}$.

Let $\varepsilon>0$ be the scale parameter labelling the family of the shot-noise fields

$$
\zeta^{(\varepsilon)}(x)=\sum_{i} \xi_{i} \varphi\left(\left(x-x_{i}^{(\varepsilon)}\right) / \theta_{i}\right), \quad x \in \mathbf{R}^{d},
$$

where $\varphi: \mathbf{R}^{d} \rightarrow \mathbf{R}$ is a nonrandom function, $\left\{x_{i}^{(\varepsilon)}\right\}$ is the Poisson point process on $\mathbf{R}^{d}$ with values in $\mathbf{R}^{d}$ and intensity function $\lambda_{\varepsilon}(x)=\lambda_{0}(\varepsilon x), x \in \mathbf{R}^{d}$, and $\left(\xi_{i}, \theta_{i}\right), i \in \mathbf{N}$, is a sequence of i.d.d. random vectors with values in $\mathbf{R} \times \mathbf{R}_{+}$having the same distribution as $(\xi, \theta)$ (here $\theta>0$ a.s.) with d.f. $G(\cdot, \cdot)$; all the random objects are considered on some probability space $(\Omega, \mathscr{F}, P)$. Further assumptions on $(\xi, \theta), \varphi(\cdot), \lambda_{0}(\cdot)$ are given below. The shot-noise fields are discussed e.g. in [7], [9], [10].

Let us denote by $Z_{t}^{(\varepsilon)}(\alpha, a), \alpha \in \mathbf{R}_{+}, a \in \mathbf{R}^{d}$, the field $Z_{t}\left(\alpha, a ; \Phi_{0}^{(\varepsilon)}, \frac{1}{2}\right)$ corresponding to $\Phi_{0}^{(\varepsilon)}(x)=-\zeta^{(\varepsilon)}(x)$, where the minus sign is used only for the sake of convenience. The behavior of $Z_{t}^{(\varepsilon)}(\alpha, a)$ will be different for time parameters with different dependence on the scale $\varepsilon$.

We deal with scalings having the properties

$$
\varepsilon \rightarrow 0, \quad t \rightarrow \infty, \quad \varepsilon \sqrt{t} \rightarrow c, \quad 0 \leq c \leq \infty .
$$

From the physical point of view it is interesting to consider a periodical function $\lambda_{0}(\cdot)$. However, in the case $0 \leq c<\infty$ we can proceed without this assumption (see [4]).

Note that a different asymptotic problem for the Burgers equation was investigated by M.S. Rosenblatt [11]. For $d=1$ and $t>0$ fixed he studied the behavior of integrals with respect to $x$ for the solution of the Burgers equation. Special attention was paid in [11] to strongly mixing initial data $v_{0}(x)$ and to the Gaussian case. It is worth emphasizing that we consider non-gaussian shot-noise fields which in general do not possess such mixing properties even for $d=1$. 
Thus, we study the vector-valued fields

$$
Z_{t}^{(\varepsilon)}(\alpha, a)=V_{t}^{(\varepsilon)}(\alpha, a) / J_{t}^{(\varepsilon)}(\alpha, a), \quad \alpha \in \mathbf{R}_{+}, a \in \mathbf{R}^{d}
$$

where

$$
\begin{aligned}
V_{t}^{(\varepsilon)}(\alpha, a) & =\int_{\mathbf{R}^{d}} \frac{a \sqrt{t}-y}{\alpha t} \exp \left\{\zeta^{(\varepsilon)}(y)-\frac{|a \sqrt{t}-y|^{2}}{2 \alpha t}\right\} d y \\
J_{t}^{(\varepsilon)}(\alpha, a) & =\int_{\mathbf{R}^{d}} \exp \left\{\zeta^{(\varepsilon)}(y)-\frac{|a \sqrt{t}-y|^{2}}{2 \alpha t}\right\} d y .
\end{aligned}
$$

The analysis of the asymptotic behavior of the fields $Z_{t}^{(\varepsilon)}$ is based on the joint study of the fields $V_{t}^{(\varepsilon)}$ and $J_{t}^{(\varepsilon)}$, various approximation schemes and limit theorems for sums of dependent multi-indexed random variables.

The main result is that (under specified conditions)

$$
\tilde{Z}_{t}^{(\varepsilon)}(\alpha, a)=\mathscr{E}_{t}^{(\varepsilon)}(\alpha, a) t^{(d+2) / 4} Z_{t}(\alpha, a)-t^{d / 4} \mathscr{M}_{t}^{(\varepsilon)}(\alpha, a) \stackrel{D}{\rightsquigarrow} Z_{c}(\alpha, a)
$$

i.e., all the finite dimensional distributions of the field $\tilde{Z}_{t}^{(\varepsilon)}$ converge weakly to the corresponding finite dimensional distributions of a vector-valued centered Gaussian field $Z_{c}$ with the covariance structure given by the matrices $T_{c}(\alpha, a ; \beta, b), \alpha, \beta \in$ $\mathbf{R}_{+} ; a, b \in \mathbf{R}^{d}$. The value of the parameter $c$ is defined by the limit condition (2). Moreover, explicit formulas are obtained for $T_{c}(\alpha, a ; \beta, b)$ and the non-random functions $\mathscr{E}_{t}^{(\varepsilon)}(\alpha, a), \mathscr{M}_{t}^{(\varepsilon)}(\alpha, a)$ taking values in $\mathbf{R}$ and $\mathbf{R}^{d}$, respectively.

We start with the following simply verified

Lemma 1. Let $h(x), M(x), x \in \mathbf{R}^{d}$, be real-valued functions such that $M(\cdot) \in L^{1}\left(\mathbf{R}^{d}\right)$ (with respect to the Lebesgue measure) and $h(\cdot)$ is continuous and periodical with a period $\left(T_{1}, \ldots, T_{d}\right)$. Then

$$
\int_{\mathbf{R}^{d}} M(x) h(\tau x) d x \rightarrow\langle h\rangle \int_{\mathbf{R}^{d}} M(x) d x \quad \text { as } \tau \rightarrow \infty,
$$

where

$$
\langle h\rangle=\left(\prod_{i=1}^{d} T_{i}\right)^{-1} \int_{0}^{T_{1}} \cdots \int_{0}^{T_{d}} h(x) d x .
$$

The next two results give us the mean values of $J_{t}^{(\varepsilon)}$ and $V_{t}^{(\varepsilon)}$. 
Lemma 2. Suppose $\varphi(\cdot) \in L^{1}\left(\mathbf{R}^{d}\right) \cap L^{\infty}\left(\mathbf{R}^{d}\right), \mathbf{E} \theta^{d} \exp \left\{\|\varphi\|_{\infty}|\xi|\right\}<\infty$ (cf. (1)), and suppose $\lambda_{0}(\cdot)$ belongs to the class $\mathrm{PC}\left(\mathbf{R}^{d}\right)$ of periodical continuous functions. Then $\mathbf{E} J_{t}^{(\varepsilon)}(\alpha, a)=t^{d / 2} \mathscr{E}_{t}^{(\varepsilon)}(\alpha, a)$ for all $\alpha \in \mathbf{R}_{+}, a \in \mathbf{R}^{d}$, where

$$
\begin{gathered}
\mathscr{E}_{t}^{(\varepsilon)}(\alpha, a)=\int_{\mathbf{R}^{d}} \exp \left\{-\frac{|y-a|^{2}}{2 \alpha}+\Lambda_{t}^{(\varepsilon)}(y)\right\} d y \\
\Lambda_{t}^{(\varepsilon)}(y)=\int_{\mathbf{R}^{d}} \int_{\mathbf{R}_{+}} \int_{\mathbf{R}} \lambda_{0}(\varepsilon z)\left(\exp \left\{v \varphi\left(\frac{y \sqrt{t}-z}{u}\right)\right\}-1\right) d G(u, v) d z .
\end{gathered}
$$

Under the scaling condition (2)

$$
\mathscr{E}_{t}^{(\varepsilon)}(\alpha, a)=\mathscr{E}_{c}(\alpha, a)+g_{t}^{(\varepsilon)}(\alpha, a), \quad \text { with } g_{t}^{(\varepsilon)}(\alpha, a) \rightarrow 0 .
$$

Here, denoting

$$
H=\int_{\mathbf{R}^{d}} \int_{\mathbf{R}_{+}} \int_{\mathbf{R}}(\exp \{v \varphi(x)\}-1) u^{d} d G(u, v) d x
$$

we have

$$
\mathscr{E}_{c}(\alpha, a)= \begin{cases}\mathscr{E}_{0}(\alpha)=(2 \pi \alpha)^{d / 2} \exp \left\{\lambda_{0}(0) H\right\}, & c=0, \\ \int_{\mathbf{R}^{d}} \exp \left\{-\frac{|y-a|^{2}}{2 \alpha}+\lambda_{0}(c y) H\right\} d y, & 0<c<\infty, \\ \mathscr{E}_{\infty}(\alpha)=(2 \pi \alpha)^{d / 2}\left\langle\exp \left\{\lambda_{0}(\cdot) H\right\}\right\rangle, & c=\infty .\end{cases}
$$

Lemma 3. Let the conditions of Lemma 2 be satisfied. Then

$$
\mathbf{E} V_{t}^{(\varepsilon)}(\alpha, a)=t^{(d-1) / 2} \mathscr{M}_{t}^{(\varepsilon)}(\alpha, a)
$$

where

$$
\begin{gathered}
\mathscr{M}_{t}^{(\varepsilon)}(\alpha, a)=-\frac{1}{\alpha} \int_{\mathbf{R}^{d}}(y-a) \exp \left\{-\frac{|y-a|^{2}}{2 \alpha}+\Lambda_{t}^{(\varepsilon)}(y)\right\} d y, \\
\mathscr{M}_{t}^{(\varepsilon)}(\alpha, a)=\mathscr{M}_{c}(\alpha, a)+h_{t}^{(\varepsilon)}(\alpha, a),
\end{gathered}
$$

and $h_{t}^{(\varepsilon)}(\alpha, a) \rightarrow 0$, provided (2) holds. Here

$$
\mathscr{M}_{c}(\alpha, a)= \begin{cases}-\frac{1}{\alpha} \int_{\mathbf{R}^{d}}(y-a) \exp \left\{\frac{|y-a|^{2}}{2 \alpha}+\lambda_{0}(c y) H\right\} d y, & 0<c<\infty, \\ 0, & c=0, c=\infty .\end{cases}
$$

Now we consider the cut-off fields

$$
\zeta_{r}^{(\varepsilon)}(x)=\sum_{i} \xi_{i} \varphi_{r}\left(\left(x-x_{i}^{(\varepsilon)}\right) / \theta_{i}\right), \quad x \in \mathbf{R}^{d},
$$

where $\varphi_{r}(x)=\varphi(x) \mathbf{1}\{|x| \leq r\}, x \in \mathbf{R}^{d}$, and $r=r(t), t>0$. Here $\mathbf{1}\{\cdot\}$ is the indicator function. Substituting $\zeta_{r}^{(\varepsilon)}$ instead of $\zeta^{(\varepsilon)}$ into the expressions (3) for $J_{t}^{(\varepsilon)}$ and $V_{t}^{(\varepsilon)}$ we obtain approximating fields $J_{t, r}^{(\varepsilon)}$ and $V_{t, r}^{(\varepsilon)}$ as the following lemma shows.

From now on the index $i$ to the left of the vector symbol denotes the $i$ th component. 
Lemma 4. Let the assumptions of Lemma 2 be strengthened by the hypothesis $\mathbf{E} \theta^{d} \exp \left\{2\|\varphi\|_{\infty}|\xi|\right\}<\infty$. Then for all $\alpha, t, \varepsilon, r \in \mathbf{R}_{+} ; a \in \mathbf{R}^{d}$, $i=1, \ldots, d$,

$$
\begin{gathered}
\mathbf{E}\left|J_{t}^{(\varepsilon)}(\alpha, a)-J_{t, r}^{(\varepsilon)}(\alpha, a)\right| \leq L(\alpha t)^{d / 2} R(\varphi, r), \\
\mathbf{E}\left|{ }_{i} V_{t}^{(\varepsilon)}(\alpha, a)-{ }_{i} V_{t, r}^{(\varepsilon)}(\alpha, a)\right| \leq L(\alpha t)^{(d-1) / 2} R(\varphi, r),
\end{gathered}
$$

where

$$
R(\varphi, r)=\left(\int_{|x|>r} \varphi^{2}(x) d x+\left(\int_{|x|>r} \varphi(x) d x\right)^{2}\right)^{1 / 2}
$$

and $L>0$ is independent of $\varepsilon, t, r, \alpha, a$.

Now we introduce the following auxiliary fields

$$
\zeta_{r, \gamma}^{(\varepsilon)}(x)=\sum_{i:\left|x-x_{i}^{(e)}\right| \leq \gamma r} \xi_{i} \varphi_{r}\left(\left(x-x_{i}^{(\varepsilon)}\right) / \theta_{i}\right), \quad x \in \mathbf{R}^{d}, \gamma>0
$$

and let $J_{t, r, \gamma}^{(\varepsilon)}(\alpha, a), V_{t, r, \gamma}^{(\varepsilon)}(\alpha, a)$ be defined according to (3) replacing $\zeta^{(\varepsilon)}$ by $\zeta_{r, \gamma}^{(\varepsilon)}$. Put for $q \in \mathbf{N}, \gamma \in \mathbf{R}_{+}$

$$
M(q, \gamma)=\max _{l=1 \ldots, q} \mathbf{E}|\xi|^{l} \theta^{d} \mathbf{1}\{\theta>\gamma\}
$$

and for $s>2$

$$
q_{0}(s)=\inf \left\{K: K \geq s(s-2)^{-1}, K \in \mathbf{N}\right\}
$$

Lemma 5. Let the assumptions of Lemma 2 be strengthened by the hypothesis $\mathbf{E} \theta^{d} \exp \left\{s\|\varphi\|_{\infty}|\xi|\right\}<\infty$ for some $s \in(2,3]$. Then for all $\alpha, t, \varepsilon, r$, $\gamma \in \mathbf{R}_{+} ; a \in \mathbf{R}^{d} ; i=1, \ldots, d$, and $q_{0}=q_{0}(s)$

$$
\begin{gathered}
\mathbf{E}\left|J_{t, r}^{(\varepsilon)}(\alpha, a)-J_{t, r, \gamma}^{(\varepsilon)}(\alpha, a)\right| \leq k(\alpha t)^{d / 2} \max \left\{M\left(q_{0}, \gamma\right), M^{1 / q_{0}}\left(q_{0}, \gamma\right)\right\}, \\
\mathbf{E}\left|{ }_{i} V_{t, r}^{(\varepsilon)}(\alpha, a)-V_{t, r, \gamma}^{(\varepsilon)}(\alpha, a)\right| \leq k(\alpha t)^{(d-1) / 2} \max \left\{M\left(q_{0}, \gamma\right), M^{1 / q_{0}}\left(q_{0}, \gamma\right)\right\},
\end{gathered}
$$

where $k>0$ is independent of $\varepsilon, t, r, \gamma, \alpha$ and $a$.

The essential part of the study of the limiting behavior of the functionals $J_{t, r, \gamma}^{(\varepsilon)}, V_{t, r, \gamma}^{(\varepsilon)}$ consists of an analysis of the covariance structure of these fields. 
Denote for $\alpha, \beta \in \mathbf{R}_{+}$and $a, b \in \mathbf{R}^{d}$

$$
\begin{aligned}
& K_{\alpha, \beta}(x ; a, b)=\exp \left\{-\frac{|x-a|^{2}}{2 \alpha}-\frac{|x-b|^{2}}{2 \beta}\right\}, \\
& K_{\alpha, \beta}(a, b)=\left(\frac{2 \pi \alpha \beta}{\alpha+\beta}\right)^{d / 2} \exp \left\{-\frac{|a-b|^{2}}{2(\alpha+\beta)}\right\} .
\end{aligned}
$$

Lemma 6. Let the conditions of Lemma 2 be strengthened by the hypothesis $\mathbf{E} \theta^{2 d} \exp \left\{2\|\varphi\|_{\infty}|\xi|\right\}<\infty$. Then for all $\alpha, \beta, t, \varepsilon, r, \gamma \in \mathbf{R}_{+} ; a, b \in \mathbf{R}^{d}$

$$
\operatorname{Cov}\left(J_{t, r, \gamma}^{(\varepsilon)}(\alpha, a), J_{t, r, \gamma}^{(\varepsilon)}(\beta, b)\right)=t^{d / 2}\left(\sigma_{c}(\alpha, a ; \beta, b)+g_{t, r, \gamma}^{(\varepsilon)}(\alpha, a ; \beta, b)\right)
$$

where

$$
\begin{gathered}
\sigma_{c}(\alpha, a ; \beta, b)= \begin{cases}K_{\alpha, \beta}(a, b) I(0), & c=0, \\
\int_{\mathbf{R}^{d}} K_{\alpha, \beta}(x ; a, b) I(c x) d x, & 0<c<\infty, \\
K_{\alpha, \beta}(a, b)\langle I(\cdot)\rangle, & c=\infty,\end{cases} \\
I(z)=\exp \left\{2 \lambda_{0}(z) H\right\} \int_{\mathbf{R}^{d}} f(z, w) d w, \\
f(z, w)=\exp \left\{\lambda _ { 0 } ( z ) \int _ { \mathbf { R } ^ { d } } \int _ { \mathbf { R } _ { + } } \int _ { \mathbf { R } } \left(\exp \left(v \varphi\left(\frac{\tau}{u}\right)-1\right) .\right.\right. \\
\left.\cdot\left(\exp \left(v \varphi\left(\frac{\tau-w}{u}\right)\right)-1\right) d G(u, v) d \tau\right\}-1,
\end{gathered}
$$

and $g_{t, r, \gamma}^{(\varepsilon)}(\alpha, a ; \beta, b) \rightarrow 0$ for each $\alpha, \beta \in \mathbf{R}_{+} ; a, b \in \mathbf{R}^{d}$ under the condition

$$
\varepsilon \rightarrow 0, \quad t \rightarrow \infty, \quad \varepsilon \sqrt{t} \rightarrow c(0 \leq c \leq \infty), \quad r(t) \rightarrow \infty, \quad \gamma(t) \geq \gamma_{0} ;
$$

here $\gamma_{0}$ is some positive constant.

The proof of this lemma is based on the following formulas:

$$
\begin{gathered}
\operatorname{Cov}\left(J_{t, r, \gamma}^{(\varepsilon)}(\alpha, a), J_{t, r, \gamma}^{(\varepsilon)}(\beta, b)\right) \\
=t^{d / 2} \int_{\mathbf{R}^{d}} K_{\alpha, \beta}(x ; a, b) H_{\varepsilon, r, \gamma}(x \sqrt{t}) D_{\varepsilon, t, r, \gamma}(x \sqrt{t} ; \beta, b) d x \\
H_{\varepsilon, r, \gamma}(y)=\exp \left\{\int_{\mathbf{R}^{d}} \int_{\mathbf{R}_{+}} \int_{\mathbf{R}^{\prime}} \lambda_{0}(\varepsilon z)\left(\exp \left(v \Psi_{r, \gamma}(y-z, u)\right)-1\right) d G(u, v) d z\right\} \\
D_{\varepsilon, t, r, \gamma}(y ; \beta, b)=\int_{\mathbf{R}^{d}} H_{\varepsilon, r, \gamma}(y-w) F_{\varepsilon, r, \gamma}(y, y-w) . \\
\cdot \exp \left\{-\frac{1}{\beta}\left(\frac{|w|^{2}}{2 t}-\frac{(y, w)}{t}+\frac{(b, w)}{\sqrt{t}}\right)\right\} d w
\end{gathered}
$$




$$
\begin{gathered}
F_{\varepsilon, r, \gamma}(x, z)=\exp \left\{\int_{\mathbf{R}^{d}} \int_{\mathbf{R}_{+}} \int_{\mathbf{R}} \lambda_{0}(\varepsilon \tau)\left(\exp \left(v \Psi_{r, \gamma}(x-\tau, u)\right)-1\right) .\right. \\
\left.\cdot\left(\exp \left(v \Psi_{r, \gamma}(z-\tau, u)\right)-1\right) d G(u, v) d \tau\right\}-1, \\
\Psi_{r, \gamma}(x, u)=\varphi_{r}\left(\frac{x}{u}\right) \mathbf{1}\{|x| \leq r \gamma\} .
\end{gathered}
$$

Making a number of estimates of the above integrals, using the fact that for all $x, b \in \mathbf{R}^{d} ; \beta, t \in \mathbf{R}_{+}$

$$
\sup _{w \in \mathbf{R}^{d}} \exp \left\{-\frac{1}{\beta}\left(\frac{|w|^{2}}{2 t}+\frac{(b, w)}{\sqrt{t}}-\frac{(x, w)}{\sqrt{t}}\right)\right\}=\exp \left\{\frac{|x-b|^{2}}{2 \beta}\right\}
$$

and Lemma 1 we can finish the proof of Lemma 6.

The next two lemmas can be established analogously by using, instead of (11), the fact that for all $x, b \in \mathbf{R}^{d} ; \beta, t \in \mathbf{R}_{+} ; i=1, \ldots, d$,

$$
\begin{gathered}
\sup _{\substack{w \in \mathbf{R}^{d} \\
w \neq 0}} \exp \left\{-\frac{1}{\beta}\left(\frac{|w|^{2}}{2 t}+\frac{(b, w)}{\sqrt{t}}-\frac{(x, w)}{\sqrt{t}}\right)+\ln \left(\frac{\left|{ }_{i} w\right|}{\sqrt{t}}\right)\right\} \\
\left.\leq\left({ }_{i} x-{ }_{i} b\right)^{2}+4 \beta\right)^{1 / 2} \exp \left\{\frac{|x-b|^{2}}{2 \beta}\right\} .
\end{gathered}
$$

Lemma 7. Suppose the conditions of Lemma 6 are satisfied. Then for all $\alpha, \beta \in \mathbf{R}_{+} ; a, b \in \mathbf{R}^{d} ; i, k=1, \ldots, d$,

$$
\operatorname{Cov}\left({ }_{i} V_{t, r}^{(\varepsilon)}(\alpha, a),{ }_{k} V_{t, r}^{(\varepsilon)}(\beta, b)\right)=t^{(d-2) / 2}\left(B_{c}^{(i, k)}(\alpha, a ; \beta, b)+H_{\varepsilon, t, r, \gamma}^{(i, k)}(\alpha, a ; \beta, b)\right),
$$

where for $0<c<\infty ; i, k=1, \ldots, d$,

$$
B_{c}^{(i, k)}(\alpha, a ; \beta, b)=\frac{1}{\alpha \beta} \int_{\mathbf{R}^{d}}\left({ }_{i} x-{ }_{i} a\right)\left({ }_{k} x-{ }_{k} b\right) K_{\alpha, \beta}(x ; a, b) I(c x) d x
$$

and for $c=0$ and $c=\infty$

$$
\begin{gathered}
B_{c}^{(i, k)}(\alpha, a ; \beta, b)=-\frac{\alpha \beta}{(\alpha+\beta)^{2}}\left({ }_{i} a-{ }_{i} b\right)\left({ }_{k} a-{ }_{k} b\right) \sigma_{c}(\alpha, a ; \beta, b), \quad \text { if } i \neq k \\
B_{c}^{(i, i)}(\alpha, a ; \beta, b)=\frac{1}{\alpha+\beta}\left(1-\frac{\left({ }_{i} a-{ }_{i} b\right)^{2}}{\alpha+\beta}\right) \sigma_{c}(\alpha, a ; \beta, b),
\end{gathered}
$$

and $H_{\varepsilon, t, r, \gamma}^{(i, k)}(\alpha, a ; \beta, b) \rightarrow 0$ under the condition (10). 
Lemma 8. Suppose the conditions of Lemma 6 are fulfilled. Then for all $\alpha, \beta \in \mathbf{R}_{+} ; a, b \in \mathbf{R}^{d} ; i=1, \ldots, d$,

$$
\operatorname{Cov}\left({ }_{i} V_{t, r, \gamma}^{(\varepsilon)}(\alpha, a), J_{t, r, \gamma}^{(\varepsilon)}(\beta, b)\right)=t^{(d-1) / 2}\left({ }_{i} \varrho_{c}(\alpha, a ; \beta, b)+{ }_{i} l_{t, r, \gamma}^{(\varepsilon)}(\alpha, a ; \beta, b)\right),
$$

where

$$
\varrho_{c}(\alpha, a ; \beta, b)= \begin{cases}-\frac{1}{\alpha} \int_{\mathbf{R}^{d}}(x-a) K_{\alpha, \beta}(x ; a, b) I(c x) d x, & 0<c<\infty, \\ \frac{\beta}{\alpha+\beta}(a-b) \sigma_{c}(\alpha, a ; \beta, b), & c=0, c=\infty,\end{cases}
$$

and $l_{t, r, \gamma}^{(\varepsilon)}(\alpha, a ; \beta, b) \rightarrow 0$ (in $\mathbf{R}^{d}$ ) under the condition (10).

The next step of approximation consists of transferring the integration over $\mathbf{R}^{d}$, in expressions for $J_{t, r, \gamma}^{(\varepsilon)}$ and $V_{t, r, \gamma}^{(\varepsilon)}$, to the integration over the cubes $Q(h)=$ $([-h, h])^{d}$ with $h=h(t)$. Thereby we introduce the fields $J_{t, r, \gamma, h}^{(\varepsilon)}$ and $V_{t, r, \gamma, h}^{(\varepsilon)}$.

Lemma 9. Let the conditions of Lemma 6 be satisfied and let $h(t) / \sqrt{t} \rightarrow \infty$ as $t \rightarrow \infty$. Then, under the condition (10), for each $\alpha \in \mathbf{R}_{+} ; a \in \mathbf{R}^{d} ; i=$ $1, \ldots, d$,

$$
\begin{gathered}
t^{-d / 2} \operatorname{Var}\left(J_{t, r, \gamma}^{(\varepsilon)}(\alpha, a)-J_{t, r, \gamma, h}^{(\varepsilon)}(\alpha, a)\right) \rightarrow 0, \\
t^{(-d+2) / 2} \operatorname{Var}\left({ }_{i} V_{t, r, \gamma}^{(\varepsilon)}(\alpha, a)-{ }_{i} V_{t, r, \gamma, h}^{(\varepsilon)}(\alpha, a)\right) \rightarrow 0 .
\end{gathered}
$$

To give an exact formulation of the limiting behavior of the normalized fields $Z_{t}^{(\varepsilon)}$ we have to introduce the following matrices

$$
A_{c}(\alpha, a ; \beta, b)=\left(A_{c}^{(i, k)}(\alpha, a ; \beta, b)\right)_{i, k=1}^{d}, \quad \alpha, \beta \in \mathbf{R}_{+} ; a, b, \in \mathbf{R}^{d} ; c \in[0, \infty],
$$

where

$$
\begin{gathered}
A_{c}^{(i, k)}(\cdot)=B_{c}^{(i, k)}(\cdot) \quad \text { for } i, k=1, \ldots, d ; \\
A_{c}^{(i, d+1)}(\alpha, a ; \beta, b)=A_{c}^{(d+1, i)}(\beta, b ; \alpha, a)={ }_{i} \varrho_{c}(\alpha, a ; \beta, b),
\end{gathered}
$$

for $i=1, \ldots, d$;

$$
A_{c}^{(d+1, d+1)}(\cdot)=\sigma_{c}(\cdot)
$$

are defined as in Lemmas 6-8.

Let $T_{c}(\alpha, a ; \beta, b)=\left(T_{c}^{(i, k)}(\alpha, a ; \beta, b)\right)_{i, k=1}^{d}$, where

$$
\begin{aligned}
& T_{c}^{(i, k)}(\alpha, a ; \beta, b)=A_{c}^{(i, k)}(\alpha, a ; \beta, b)-{ }_{i} \mathscr{L}_{c}(\alpha, a) A_{c}^{(d+1, k)}(\alpha, a ; \beta, b) \\
& -{ }_{k} \mathscr{L}_{c}(\beta, b) A_{c}^{(i, d+1)}(\alpha, a ; \beta, b)+{ }_{i} \mathscr{L}_{c}(\alpha, a){ }_{k} \mathscr{L}_{c}(\beta, b) A_{c}^{(d+1, d+1)}(\alpha, a ; \beta, b),
\end{aligned}
$$

and

$$
\mathscr{L}_{c}(\alpha, a)=\mathscr{E}_{c}^{-1}(\alpha, a) \mathscr{M}_{c}(\alpha, a) .
$$

The functions $\mathscr{E}_{c}(\cdot)$ and $\mathscr{M}_{c}(\cdot)$ have been introduced in Lemmas 2 and 3. 
Remark 1. For $c=0$ and $c=\infty$ we have for all $\alpha, \beta \in \mathbf{R}_{+} ; a, b \in \mathbf{R}^{d}$

$$
T_{c}(\alpha, a ; \beta, b)=B_{c}(\alpha, a ; \beta, b) .
$$

If $\lambda_{0}(x) \equiv$ const. then $\mathscr{E}_{t}^{(\varepsilon)}(\alpha, a)=\mathscr{E}_{0}(\alpha), \mathscr{M}_{t}^{(\varepsilon)}(\alpha, a)=0$ for all $\alpha \in \mathbf{R}, a \in \mathbf{R}^{d}$, and $B_{c}^{(i, k)}(\cdot), i, k=1, \ldots, d$, are given by (12) and (13).

Theorem 1. Let $\lambda_{0}(\cdot) \in \mathrm{PC}\left(\mathbf{R}^{d}\right)$, let $\varphi(\cdot) \in L^{1}\left(\mathbf{R}^{d}\right) \cap L^{\infty}\left(\mathbf{R}^{d}\right)$ and $\mathbf{E} \theta^{2 d} \exp \left\{s\|\varphi\|_{\infty}|\xi|\right\}<\infty$ for some $s \in(2,3]$. Assume there exist functions $r(t), \gamma(t), t>0$, satisfying

$$
\begin{gathered}
M\left(q_{0}, \gamma(t)\right)=o\left(t^{-d q_{0} / 4}\right) \quad \text { for } q_{0}=q_{0}(s), \\
r(t) \rightarrow \infty, \quad r(t) \gamma(t)=o\left(t^{\kappa}\right), \quad \kappa=\frac{1}{4}(s-2)(s-1)^{-1},
\end{gathered}
$$

and

$$
R(\varphi, r(t))=o\left(t^{-d / 4}\right) \quad \text { as } t \rightarrow \infty
$$

Then under the limit condition (2) the relation (4) is valid with $\mathscr{E}_{t}^{(\varepsilon)}$ and $\mathscr{M}_{t}^{(\varepsilon)}$ defined by (5) and (6), and the covariance matrices $T_{c}$ are given by (14) $\left(M\left(q_{0}, \gamma\right)\right.$, $q_{0}(s)$ and $R(\varphi, r)$ were introduced by (8), (9), and (7), respectively).

We indicate the main steps of the proof. At first, it is not difficult to see that for all $\alpha, \varepsilon, t \in \mathbf{R}_{+} ; a \in \mathbf{R}^{d}$

$$
\tilde{Z}_{t}^{(\varepsilon)}(\alpha, a)=\xi_{t}^{(\varepsilon)}(\alpha, a)-\mathscr{L}_{c}(\alpha, a) \eta_{t}^{(\varepsilon)}(\alpha, a)+\Delta_{t}^{(\varepsilon)}(\alpha, a),
$$

where

$$
\begin{gathered}
\xi_{t}^{(\varepsilon)}(\cdot)=t^{-(d+2) / 4}\left(V_{t}^{(\varepsilon)}(\cdot)-\mathbf{E} V_{t}^{(\varepsilon)}(\cdot)\right), \\
\eta_{t}^{(\varepsilon)}(\cdot)=t^{-d / 4}\left(J_{t}^{(\varepsilon)}(\cdot)-\mathbf{E} J_{t}^{(\varepsilon)}(\cdot)\right), \\
\Delta_{t}^{(\varepsilon)}(\cdot)=-t^{-d / 4} \eta_{t}^{(\varepsilon)}(\cdot)\left(\mathscr{E}_{t}^{(\varepsilon)}(\cdot)+t^{-d / 4} \eta_{t}^{(\varepsilon)}(\cdot)\right)^{-1} \\
\cdot\left\{\xi_{t}^{(\varepsilon)}(\cdot)-\left(\mathscr{E}_{t}^{(\varepsilon)}(\cdot)\right)^{-1} \mathscr{M}_{t}^{(\varepsilon)}(\cdot) \eta_{t}^{(\varepsilon)}(\cdot)\right\} \\
+\left\{\mathscr{L}_{c}(\cdot)-\left(\mathscr{E}_{t}^{(\varepsilon)}(\cdot)\right)^{-1} \mathscr{M}_{t}^{(\varepsilon)}(\cdot)\right\} \eta_{t}^{(\varepsilon)}(\cdot),
\end{gathered}
$$

and the vector $\mathscr{L}_{c}(\cdot)$ is defined by (16). Next, one verifies that

$$
\left(\xi_{t}^{(\varepsilon)}(\cdot), \eta_{t}^{(\varepsilon)}(\cdot)\right) \stackrel{D}{\rightsquigarrow}\left(\xi_{c}(\cdot), \eta_{c}(\cdot)\right)
$$


where $\left(\xi_{c}(\alpha, a), \eta_{c}(\alpha, a)\right)$ is a $(d+1)$-dimensional centered Gaussian field on $\mathbf{R}_{+} \times$ $\mathbf{R}^{d}$ with a covariance structure given by $A_{c}(\alpha, a ; \beta, b)$, see (14).

Using the previous lemmas and well-known properties of weak convergence and convergence in probability it suffices to show that (10) and $h(t) / \sqrt{t} \rightarrow \infty$ imply

$$
\left(\xi_{t, r, \gamma, h}^{(\varepsilon)}(\cdot), \eta_{t, r, \gamma, h}^{(\varepsilon)}(\cdot)\right) \stackrel{D}{\rightsquigarrow}\left(\xi_{c}(\cdot), \eta_{c}(\cdot)\right) .
$$

Here

$$
\begin{gathered}
\xi_{t, r, \gamma, h}^{(\varepsilon)}(\cdot)=t^{-(d+2) / 4}\left(V_{t, r, \gamma, h}^{(\varepsilon)}(\cdot)-\mathbf{E} V_{t, r, \gamma, h}^{(\varepsilon)}(\cdot)\right), \\
\eta_{t, r, \gamma, h}^{(\varepsilon)}(\cdot)=t^{-d / 4}\left(J_{t, r, \gamma, h}^{(\varepsilon)}(\cdot)-\mathbf{E} J_{t, r, \gamma, h}^{(\varepsilon)}(\cdot)\right) .
\end{gathered}
$$

We can represent $Q(h)$ as a union of "unit" cubes. Let $h(t) \in \mathbf{N}$, then $Q(h)=\cup_{j \in T(h)} K_{j}, K_{j}=\left(j_{1}-1, j_{1}\right] \times \cdots \times\left(j_{d}-1, j_{d}\right], T(h) \subset \mathbf{Z}^{d}$.

For each $n \in \mathbf{N}$ let us consider arbitrary fixed $\alpha_{p} \in \mathbf{R}_{+} ; a_{p} \in \mathbf{R}^{d} ; c_{p} \in \mathbf{R}^{d+1}$; $p=1, \ldots, n$. Then our problem is reduced to the CLT for the multi-indexed sums

$$
S_{t, r, \gamma, h}^{(\varepsilon)}=t^{-d / 4} \sum_{j \in T(h)}\left(X_{j}(\varepsilon, t, r, \gamma)-\mathbf{E} X_{j}(\varepsilon, t, r, \gamma)\right)
$$

where

$$
\begin{gathered}
X_{j}(\varepsilon, t, r, \gamma)=\int_{K_{j}} \exp \left\{\zeta_{r, \gamma}^{(\varepsilon)}(y)\right\} \Psi(y, t) d y, \quad j \in T(h), \\
\Psi(y, t)=\sum_{p=1}^{n}\left({ }_{d+1} c_{p}+\sum_{i=1}^{d}{ }_{i} c_{p} \frac{\left({ }_{i} a_{p} \sqrt{t}-{ }_{i} y\right)}{\alpha_{p} \sqrt{t}}\right) \exp \left\{-\frac{\left|a_{p} \sqrt{t}-y\right|^{2}}{2 \alpha_{p} t}\right\}, \quad t>0, y \in \mathbf{R}^{d} .
\end{gathered}
$$

Under the conditions $(10)$ and $h(t) / \sqrt{t} \rightarrow \infty$ we have

$$
\operatorname{Var}\left(\sum_{j \in T(h)} X_{j}(\varepsilon, t, r, \gamma)\right) \sim b^{2}(c) t^{d / 2}
$$

The dependence of $b^{2}(c)$ on $\alpha_{p}, a_{p}, c_{p}, p=1, \ldots, n$ (see Lemmas 6-8) is not indicated. It is enough to consider just the nontrivial case $b^{2}(c) \neq 0$. Note that the sums $\sum_{j \in T(h)} X_{j}(\varepsilon, t, r, \gamma)$ display irregular growth of variances (i.e. nonlinear dependence of the variance on the number of summands because $h(t) / \sqrt{t} \rightarrow \infty$ as $t \rightarrow \infty)$. Note also that the fields $X_{j}(\varepsilon, t, r, \gamma)$ are not stationary.

The field $X_{j}(\varepsilon, t, r, \gamma)$ is $m(t)$-dependent on the set $T(h(t))$ where $m(t)=$ $2 \gamma(t) r(t)+\sqrt{d}$. So using the CLT for the series of $m(t)$-dependent fields on $T(h(t)) \subset \mathbf{Z}^{d}$ (see [2], [5]), taking into account (20), the bound for

$$
C_{s}(\varepsilon, t, r, \gamma, h)=\max _{j \in T(h)}\left(\mathbf{E}\left|X_{j}(\varepsilon, t, r, \gamma)\right|^{s}\right)^{1 / s}, \quad s \in(2,3],
$$

the condition (18) and also the facts that $\Delta_{t}^{(\varepsilon)}(\alpha, a) \stackrel{P}{\rightarrow} 0$ for every $\alpha \in \mathbf{R}_{+}, a \in \mathbf{R}^{d}$ and $\mathscr{E}_{t}^{(\varepsilon)}(\alpha, a) \rightarrow \mathscr{E}_{c}(\alpha, a), \mathscr{M}_{t}^{(\varepsilon)}(\alpha, a) \rightarrow \mathscr{M}_{c}(\alpha, a)$ (see Lemmas 2, 3) under (2), we come to the statements of Theorem 1 . 
Corollary 1. Suppose $\lambda_{0}(\cdot) \in \mathrm{PC}\left(\mathbf{R}^{d}\right)$ and

$$
|\varphi(x)| \leq c_{0}\left(1+|x|^{d+\delta}\right)^{-1}
$$

for all $x \in \mathbf{R}^{d}$ and some $c_{0}, \delta>0$, and suppose $\mathbf{E} \theta^{2 d} \exp \left\{s\|\varphi\|_{\infty}|\xi|\right\}<\infty$ for some $s \in(2,3]$. If (17) is satisfied with $\gamma(t)=c_{1} t^{\tau}$ for some $\tau \in\left[0, \frac{1}{4}(s-2)\right.$. $\left.\cdot(s-1)^{-1}\right), c_{1}>0$, then the statements of Theorem 1 (referred in the sequel as (4)) are valid if $\delta>d\left((s-2)(s-1)^{-1}-4 \tau\right)^{-1}$. In particular, we can choose $\tau=0$ whenever $\theta \leq \theta_{0}$ a.s. for some positive constant $\theta_{0}$.

Corollary 2. Let $\lambda_{0}(\cdot) \in \mathrm{PC}\left(\mathbf{R}^{d}\right)$ and let $\varphi(\cdot) \in L^{1}\left(\mathbf{R}^{d}\right) \cap L^{\infty}\left(\mathbf{R}^{d}\right)$. Assume that for some $s \in(2,3]$ there exists a function $r(t), t>0$, with the following properties

$$
r(t) \rightarrow \infty, \quad R(\varphi, r(t))=o\left(t^{-d / 4}\right), \quad r(t)=o\left(t^{\kappa}\right) \quad \text { as } t \rightarrow \infty
$$

and $\kappa=\frac{1}{4}(s-2)(s-1)^{-1}$. If, in addition, $\mathbf{E} \exp \left\{s\|\varphi\|_{\infty}|\xi|\right\}<\infty$ and $\theta \leq \theta_{0}$ a.s., then (4) holds.

Using the technique of cumulants (see Corollary 7.3 in [5]) and strengthening the restrictions on the amplitudes $\xi_{i}$ we can relax the requirements on the function $\varphi: \mathbf{R}^{d} \rightarrow \mathbf{R}$.

Theorem 2. Let $\lambda_{0}(\cdot) \in \mathrm{PC}\left(\mathbf{R}^{d}\right), \varphi \in L^{1}\left(\mathbf{R}^{d}\right) \cap L^{\infty}\left(\mathbf{R}^{d}\right)$ and $\mathbf{E} \theta^{2 d} \exp \{\mu|\xi|\}<\infty$ for all $\mu>0$. If the conditions (19) and, respectively, (17) for $q_{0}=2$ are satisfied with $r(t) \gamma(t)=o\left(t^{\tau}\right)$ as $t \rightarrow \infty$ for some $\tau<\frac{1}{4}$, then (4) is valid.

Remark 2. This result can be considered as the limiting case of Theorem 1 as $s \rightarrow \infty$.

Corollary 3. Let $\lambda_{0}(\cdot) \in \mathrm{PC}\left(\mathbf{R}^{d}\right), \mathbf{E} \exp \{\mu \theta\}<\infty, \mathbf{E} \exp \{\mu|\xi|\}<\infty$ for all $\mu>0$ and let

$$
R\left(\varphi, t^{\tau}\right)=o\left(t^{-d / 4}\right) \quad \text { for some } \tau<\frac{1}{4} .
$$

Then (4) is true. Furthermore, instead of (23) we can assume that (21) holds for some $c_{0}>0$ and $\delta>d$.

Remark 3. In [6] a single field $\zeta(\cdot)$ of the type (1) with a Poisson point field $\left\{x_{i}\right\}$ having the intensity function $\lambda \equiv$ const. was considered and the limiting behavior as $t \rightarrow \infty$ of the field $\tilde{Z}_{t}(1, a)=\mathscr{E}_{0}(1) t^{(d+2) / 4} Z_{t}(1, a), a \in \mathbf{R}^{d}$ (i.e. $\alpha=1$ ) was established (see Remark 1). In [6] also the independence of $\left\{\xi_{i}\right\}$ and $\left\{\theta_{i}\right\}$ was supposed. The last hypothesis was used also in [3], [4] where the limiting behavior of $\tilde{Z}_{t}^{(\varepsilon)}(1, a)$ was investigated under the scaling condition (2) with $0 \leq c<\infty$. Thus the results of [3], [4], [6] can be obtained from the results in the present paper.

The author is grateful to Professor S.A. Molchanov for posing the problem as well as indicating the effect of averaging (Lemma 1). 


\section{References}

[1] Albeverio, S., and S.A. Molchanov: The net structure of the Big Universe and the Burgers equation, a probabalistic approach. - To appear.

[2] BulinskiI, A.V.: Limit theorems under weak dependence conditions. - Probability Theory and Mathematical Statistics. Proceedings of the Fourth Vilnius Conference, Volume 1, Mokslas/VSP BV, Vilnius/Utrecht, 1987, 307-326.

[3] BulinskiI, A.V.: Asymptotic normality for the families of integral functionals. - Proceedings of the fifth International Vilnius Conference on Probability and Mathematical Statistics. Abstracts of Communications 1, Vilnius, 1989, 83-84.

[4] BulinskiI, A.V.: CLT for families of integral functionals arising in solving the multidimensional Burgers equation. - Probability Theory and Mathematical Statististics. Proceedings of the Fifth Vilnius Conference, Volume 1, Mokslas/VSP BV, Vilnius/Utrecht, 1990, 207-216.

[5] Bulinskil, A.V.: Limit theorems under weak dependence conditions. - Moscow State University Press, Moscow, 1989 (Russian).

[6] Bulinskil, A.V., and S.A. Molchanov: Asymptotic normality of a solution of Burgers equation with random initial conditions. - Teor. Veroyatnost. i Primenen. 36, 1991, 217-235 (Russian).

[7] Giraitis, L., and D. Surgailis: On shot noise processes with long range dependence. Probability Theory and Mathematical Statististics. Proceedings of the Fifth Vilnius Conference, Volume 1, Mokslas/VSP BV, Vilnius/Utrecht, 1990, 401-408.

[8] Gurbatov, S.N., et al.: The large-scale structure of the Universe in the frame of the model equation of non-linear diffusion. - Preprint MPA-371, Max Planck Institut für Physik und Astrophysik, 1988, 1-21.

[9] Heinrich, L., and V. Schmidt: Normal convergence of multidimensional shot noise and rates of this convergence. - Adv. in Appl. Probab. 17, 1985, 709-730.

[10] Rice, J.: On generalized shot-noise. - Adv. in Appl. Probab. 9, 1977, 553-565.

[11] Rosenblatt, M.S.: Scale renormalization and random solutions of the Burgers equation. - J. Appl. Probab. 24, 1987, 328-338.

[12] Shandarin, S.F., and Ya.B. Zeldovich: Turbulence, intermittency, structures in a selfgravitating medium: the large-scale structure of the Universe. - Preprint MPA-350, Max Planck Institut für Physik und Astrophysik, 1988, 1-92. 